\title{
A Semantic Tag Stock For Content-oriented Image Annotation
}

\author{
Chi Zhang \\ School of Computer Science \\ Communication University of China \\ Beijing, P.R. China \\ zhangchi@cuc.edu.cn
}

\author{
PengZhou Zhang, YuJun Wen \\ School of Computer Science \\ Communication University of China \\ Beijing, P.R. China \\ zhangpengzhou@cuc.edu.cn
}

\begin{abstract}
-it is fundamental and crucial to describe digital images reasonably and effectively for image applications. In this paper, we will briefly discuss some important issues about image annotation and then present a semantic tag stock in details. The tag stock is designed to support content-oriented image annotation system with tagging technique. It has been put into practice in a large-scale image application system, and it is demonstrated to be effective and worthy of spreading.
\end{abstract}

Keywords: semantic tag; image annotation; content-oriented

\section{INTRODUCTION}

Digital images, as one of the important kinds of multimedia, have been used widely in all kinds of industries. Many large-scale image stocks have been constructed. Because image description is fundamental to image retrieval and reuse, many academic organizations and business companies have carried out a large number of studies about image metadata, and published several standards about it. However, these image metadata standards are not enough to describe the content of image, while the image content is carrying a mass of valuable information which is the substantial characteristics of itself.

The success of tagging services, like 500px ${ }^{1}$, Flickr ${ }^{2}$, has shown that tagging is a great collaboration tool. People annotate images, videos and other resources with a couple of keywords which are free text. It seems to be a natural way to describe and classify resources as well as an attractive way to discover new material. However, looking for information in the free tag space has many hard limitations. The difficulty comes from the fact that several people maybe use different tags for the same object [1]. So, to construct a controlled vocabulary, from which some useful controlled tags can be taken, is beneficial to cope with the difficulty. Many existing controlled vocabularies (CV) are either focusing on different special fields or covering the whole knowledge domain, so it is not feasible to directly reference existing CV to support tagging digital images.

This paper will focus on how to construct a structured stock, which maintains a great number of semantic tags, to support annotating the content of digital images. There are several objectives of it.

1) Be capable of expressing sufficient semantic information of controlled tags.

2) Has reasonable structure to enhance the user ${ }^{1}$ http://500px.com/

${ }^{2}$ http:www.flickr.com. experience of annotating and searching images.

3) It has good mechanisms to extend the number as well as enrich the semantic information of tags.

\section{PRELIMINARY WORK}

As mentioned above, the content of image has rich and valuable information, meanwhile it is not enough to describe it by those existing metadata specifications (such as IPTC Photo Metadata [2], Dublin core metadata [3]). Therefore, we propose to induce and specify content-oriented tagging dimensions in advance of constructing controlled tag stock. In fact, the tag stock is built based on the specification of tagging dimensions.

Furthermore, we need a moderate scale vocabulary to initialize the tag stock. It needs the effort of experts from related fields, and referring or importing some words from existing controlled vocabularies selectively. It is to be noted that the initial vocabulary could not reference $\mathrm{CV}$ or thesaurus directly. It isn't an effective method because the tags we need will meet the requirement of tagging, which is conforming to the dimensions defined in advance. Some examples are shown later.

\section{STRUCTURE OF SEMANTIC TAG STOCK}

After define the tagging dimensions of describing the content of image, we need to design the structure of tag stock. It can store and manage all of the controlled tags, and provide effective services for image annotation and retrieval. The tag stock can be implemented by some relational database.

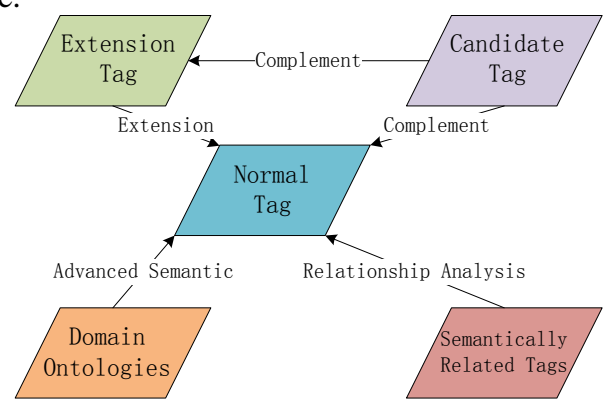

Figure 1. The Basic Structure of Tag Stock

As is shown in figure 1, the controlled tags are classified into several types: Normal Tag (NT), Extension Tag (ET), and Candidate Tag (CT). To get and maintain the semantic information of deeper level, we will analyze the relationships 
among the normal tags and create domain ontologies step by step, both of which can extend and deepen the semantic information of this tag stock.

\section{A. Normal Tag}

Normal Tags are those standard and normal words which have been examined and approved by tag administrators.

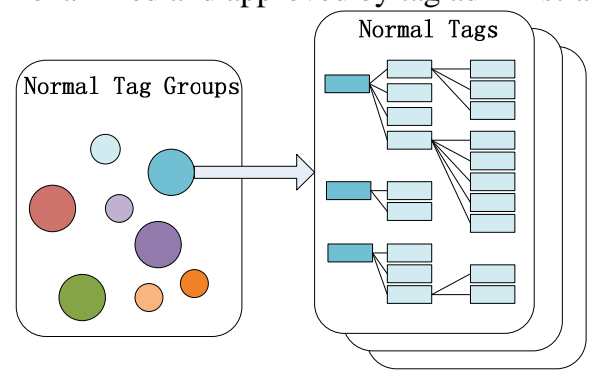

Figure 2. Structure of Normal Tag

We can define multiple normal tag groups in view of the definition of tagging dimensions. Normal tags will be grouped into tag groups through a normalization workflow, including collection, screening and confirmation. Each normal tag is regarded as a node of the tag trees within every tag group.

For example, let's assume there is a tagging dimension named "People", it means we need to fill the metadata about People to images. It is necessary to create a related tag group, which maybe includes several normal tag set: number, age, relation, gender, expression, behavior, posture, and so on. Furthermore, it is possible to construct a massive library, which collects numerous names of celebrities, to annotate editorial images.

Table 1. Key Fields of Normal Tag

\begin{tabular}{|c|l|}
\hline Field Name & \multicolumn{1}{c|}{ Definition } \\
\hline Tag Name & $\begin{array}{l}\text { A normal word which can express the meaning of } \\
\text { the tag correctly. (Required) }\end{array}$ \\
\hline $\begin{array}{c}\text { Tag } \\
\text { Description }\end{array}$ & $\begin{array}{l}\text { A textual description used to explain the meaning } \\
\text { of the tag unambiguously. (Optional) }\end{array}$ \\
\hline Tag Code & $\begin{array}{l}\text { A regular string which is unique in the tag stock. It } \\
\text { can be an identity of the tag. If a tag has multiple } \\
\text { meanings, it will emerge more than one time in the } \\
\text { tag stock and be assigned different TagCodes. } \\
\text { (Required) }\end{array}$ \\
\hline Tagging Code & $\begin{array}{l}\text { A regular string. Tagging Code of the tag is used to } \\
\text { be linked to images which are tagged by it. If } \\
\text { different tags have a same meaning, they will be } \\
\text { assigned the same TaggingCode. (Required) }\end{array}$ \\
\hline Status & $\begin{array}{l}\text { Enumeration type. The possible value range of it is } \\
\text { 'Available', 'Disable', 'Undetermined', etc. } \\
\text { (Required) }\end{array}$ \\
\hline URIs & $\begin{array}{l}\text { To record some URIs and those existing controlled } \\
\text { vocabularies or thesaurus they belong to. (Optional) }\end{array}$ \\
\hline
\end{tabular}

The details of normal tag have shown in table 1. We can distinguish the ambiguity from synonym and polysemy by the field of 'Tag Code' and 'Tagging Code'. The field of 'URIs' can link a tag to a standard word or concept published in some existing controlled vocabularies, and then do benefit to exchange metadata among different applications.

It is to be noted that only normal tag is used to tag images and the 'Tagging Code' is linked to images instead of the 'Tag Name'.

\section{B. Extension Tag}

It is required that only standard and rigorous tags could be added to the normal tag set. Meanwhile, this requirement will result in a hard limitation of tagging or searching images, because some words, which a user wants to tag, perhaps have not exist in the normal tag set, even though their meanings are similar to some normal tags. To ease off this contradiction, we create extension tag set.

Extension tags come from free words and are regarded as a complement to the normal tag. We need extension tags in the following cases: a normal tag has several bynames; many words have similar meaning, but all of them are not fit to be the normal tag.

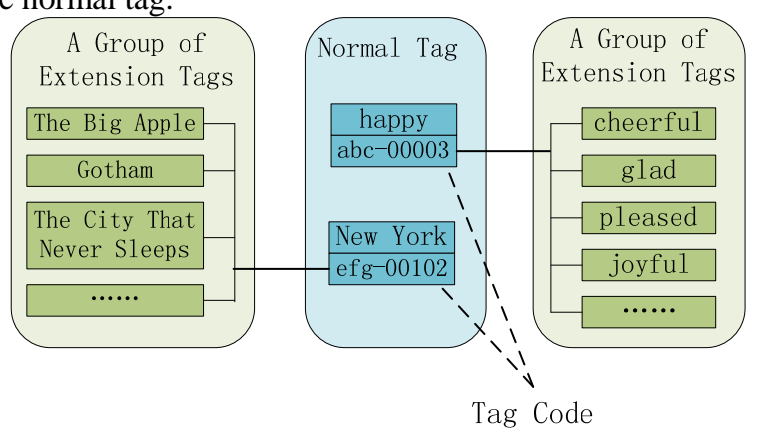

Figure 3. Examples of Extension Tag

A normal tag can be linked to a group of extension tags by the 'Tag Code' of it. When a user inputs some words and submits a request for image retrieval, the system can compare the words with not only normal tags but also extension tags. No matter which tag matches, we can get the correct 'Tag Code' and return images tagged by the code. There is a same case when we tag images.

\section{Candidate Tag}

The tag stock needs gradual extension and enrichment. The candidate tag is a necessary component to fulfill it. Candidate tags, also named temporary tags, come from those words that are used by user to either tag or search images and have not been included by the tag stock until now. The tag administrator has several choices to treat them: (1) delete them directly; (2) convert them into extension tags; (3) convert them into normal tags.

\section{SEMANTIC ANALYSIS OF DEEP LEVEL}

The tag stock can express and maintain rich semantic information. We divide it into three levels, as is shown in figure 4. 


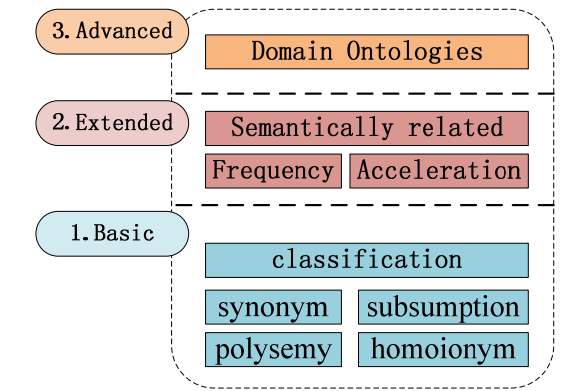

Figure 4. Semantic information of the tag stock

The basic semantic information has been specified by the normal and extension tag library mentioned in last section. Below we will explain how to obtain and make use of semantic information of deep level.

\section{A. Semantically Related Tags}

Data clustering is a common technique for statistical data analysis. Tag clustering, which is based on tag co-occurrence, is widely used to analyze the semantic relationships among free tags. Co-occurrence means tags are used for the same resource. Many methods of co-occurrence analysis have been practiced and promoted folksonomy's applications greatly [1][4][5].

There are three kinds of semantic relationship of level 2. The first is semantically related tags which can be calculated by co-occurrence analysis algorithms. The second is how many times tags have been used to either tag or search images per unit time, such as a month or a week. The third is the acceleration of frequency of using some tags to search images.

All of the semantic information mentioned above is the basis of tag recommendation which can be used in the cases of tagging and searching.

\section{B. Domain Ontologies}

Ontologies are used to capture knowledge about some domain of interest. An ontology describe the concepts in the domain and also the relationships that hold between those concepts. OWL (a standard ontology language from W3C) has a richer set of operators. Complex concepts can therefore be built up in definitions out of simpler concepts [6].

The construction of domain ontology would be completed step by step, so the relationship between ontology and tag library is designed to be loosely-coupled. That means the tag stock will still work well without any ontology, meanwhile, after an ontology is finished, it can be added to the tag stock smoothly.

Domain ontologies, which carry a large number of advanced semantic information in some domain of interest, can help us distinguish the exact meanings of tags, as well as obtain a tag set through knowledge reasoning. The reasoning rules are defined by us, which can complement the basic and extended semantic information (see figure 4).

\section{USE OF THE TAG STOCK}

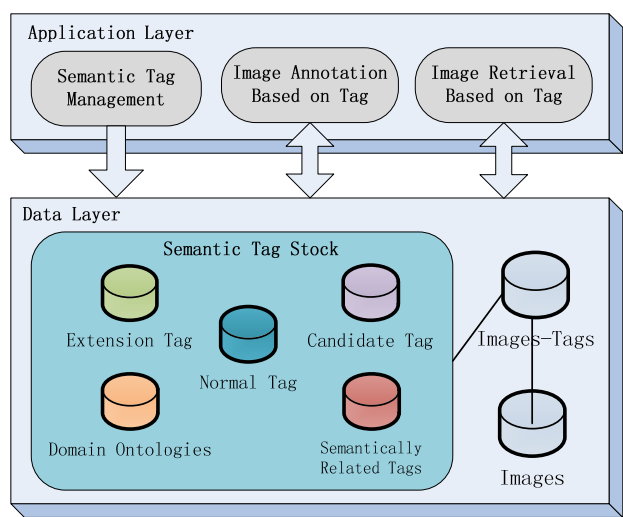

Figure 5. Architecture of tag stock application

The tag stock stated in this paper has been used in an online image stock. The architecture of it is shown in figure 5.

The semantic tag management system, shown in figure 5 , can help administrators insert and update normal and extension tags, and treat the candidate tags. Furthermore, it runs lots of services of data analysis.

Based on this tag stock, the precision ratio of image retrieval and the quality of image annotation are both improved greatly.

\section{CONCLUSIONS}

Corresponding to the objectives of the tag stock proposed in the first section, we make the conclusions as follows:

1) The tag stock has rich semantic information which can be abstracted to three levels: basic, extended and advanced.

2) The basic components of the tag stock are normal tag, extension tag and candidate tag. To specify the normal tag group and reduce the quantity of normal tags as small as possible, can do great benefit to tag images. Meanwhile, the extension tag library should have tags as many as possible, covering most of words probably used to search images. It will enhance the user experience of annotating and searching images greatly. Furthermore, the semantically related tags and domain ontologies can both contribute to tag recommendation for image retrieval and annotation.

3) Candidate tags are a main data source of the tag stock. Some external vocabularies can also imported in bulk and be normal or extension tags after approved. Semantic information will be enriched by data analysis and extending domain ontologies gradually.

There are also some problems to be resolved step by step. They include: how to fill the tag stock more efficiently, how to tag images more automatically, how to improve the efficiency of tag recommendation through the knowledge reasoning based on domain ontologies.

\section{ACKNOWLEDGMENT}

Thanks for sponsor of research fund project for young teacher of Communication University of China, and project of National Key Technology R\&D Program. 


\section{REFERENCES}

[1] Grigory Begelman, Automated Tag Clustering:Improving search and exploration in the tag space [C] / /WWW Collaborative Web Tagging Workshop, 2006.

[2] IPTC Standard Photo Metadata, www,iptc.org.

[3] DCMI Metadata Terms, http://purl.org/dc/elements/1.1/.

[4] Wang Ping, A Clustering Algorithm of Social Tags, Computer Applications and Software, Feb 2010 , in Chinese.

[5] Wu X, Zhang L, Yu Y. Exploring Social Annotations for the Semantic Web [C] / / Proceedings of the 15th international conference on World Wide Web. Edinburgh, Scotland, 2006.

[6] A Practical Guide To Building OWL Ontologies Using Protégé 4 and CO-ODE Tools. The University of Manchester. Mar, 2009 